\title{
Comparative transcriptome analysis of the lichen-forming fungus Endocarpon pusillum elucidates its drought adaptation mechanisms
}

\author{
WANG YanYan ${ }^{1,2}$, ZHANG XinYu $^{1}$, ZHOU QiMing $^{1}$, ZHANG XiaoLing ${ }^{1}$ \\ \& WEI JiangChun ${ }^{1,3 *}$ \\ ${ }^{1}$ State Key Laboratory of Mycology, Institute of Microbiology, Chinese Academy of Sciences, Beijing 100101, China; \\ ${ }^{2}$ University of Chinese Academy of Sciences, Beijing 100049, China; \\ ${ }^{3}$ State Key Laboratory of Crop Stress Biology for Arid Areas, Northwest A\&F University, Yangling 712100, China
}

Received March 21, 2014; accepted June 6, 2014; published online December 4, 2014

\begin{abstract}
The lichen-forming fungus was isolated from the desert lichen Endocarpon pusillum that is extremely drought resistant. To understand the molecular mechanisms of drought resistance in the fungus, we employed RNA-seq and quantitative real-time PCR to compare and characterize the differentially expressed genes in pure culture at two different water levels and with that in desiccated lichen. The comparative transcriptome analysis indicated that a total of 1781 genes were differentially expressed between samples cultured under normal and PEG-induced drought stress conditions. Similar to those in drought resistance plants and non-lichenized fungi, the common drought-resistant mechanisms were differentially expressed in E. pusillum. However, the expression change of genes involved in osmotic regulation in E. pusillum is different, which might be the evidence for the feature of drought adaptation. Interestingly, different from other organisms, some genes involved in drought adaption mechanisms showed significantly different expression patterns between the presence and absence of drought stress in E. pusillum. The expression of 23 candidate stress responsive genes was further confirmed by quantitative real-time PCR using dehydrated E. pusillum lichen thalli. This study provides a valuable resource for future research on lichen-forming fungi and shall facilitate future functional studies of the specific genes related to drought resistance.
\end{abstract}

lichen, mycobiont, dehydration, drought adaption, drought resistant

Citation: Wang YY, Zhang XY, Zhou QM, Zhang XL, Wei JC. Comparative transcriptome analysis of the lichen-forming fungus Endocarpon pusillum elucidates its drought adaptation mechanisms. Sci China Life Sci, 2014, 57: 89-100, doi: 10.1007/s11427-014-4760-9

Lichens are symbiotic products between fungi (the mycobionts) and photosynthetic partners (the photobionts). Each lichen species is made up of one mycobiont and at least one photobiont, and the photosynthetic partner can be either an alga or a cyanobacterium. Lichens are widely distributed across many types of ecological niches, ranging from cold to hot deserts and other extreme habitats. Unlike vascular plants, lichens lack active mechanisms for controlling water content. As a result, their water content tends to fluctuate

*Corresponding author (email: weijc2004@126.com) widely based on water availability in the environment [1]. Indeed, lichens can lose water rapidly when the environment is dry and they can also rapidly recover to normal water content when water becomes available [2]. Specifically, different from most plants, a low water content is typically nonlethal to lichens and most lichens can withstand drying to water contents of $5 \%$ or less for a long period of time $[3,4]$. Furthermore, in the presence of water, not only does the water content recover, lichens can rapidly return to normal physiological state to carry out photosynthesis and respiration during rehydration [5,6]. As a previous study 
indicated, even in a low water content state, some lichens could maintain active metabolism [7].

A number of studies have examined the effects of water availability on the morphology, physiology, and survival of lichens [1,6,8-13]. However, little is known about the molecular mechanisms of drought resistance in lichen. Many recent studies have examined the molecular mechanisms of drought resistance in plants and non-lichenized fungi. Whether similar mechanisms are involved in lichens remains unknown. In plants and non-lichenized fungi, desiccation can induce several cellular stresses, such as hyperosmolarity, hyperoxidation, hyper-ionicity and protein misfolding and aggregation [14]. To survive the desiccation, the organisms need to deal with these cellular damages. Three major stress-response pathways are known to be involved in protection from these stresses, including osmoregulation (to modulate intracellular ion concentration); DNA and protein damage repair (to prevent DNA damage and protein misfolding and degradation); and antioxidation (to scavenge reactive oxygen species (ROS)) $[15,16]$. Our limited understanding of the molecular mechanisms of drought resistance in lichens has been mainly due to the lack of genetic tools for analyzing lichens. However, recent advances in genomics technologies and the information of whole-genomes and transcriptomes from lichen-forming fungi and their photosynthetic partners are making the studies of drought resistance in lichens feasible [17,18].

Since lichen is the symbiotic product of two interacting partners, most of its biological characteristics are the result of interaction between the symbiotic partners. However, in drought resistance, the mycobiont partner seems to be the main contributor in lichens [19-21], probably due to the fact that within lichens, the photobionts are housed by the fungal tissues, which effectively protect their photosynthetic partners from desert climate. For example, a previous study indicated that, separately, the lichen-forming fungus Endocarpon pusillum has a much stronger drought resistant ability than its algal partner Diplosphaera chodatii [22]. Therefore, to understand the mechanisms of drought resistance in lichens, it is reasonable to focus on the fungal partner.

In the present study, the transcriptomes of the lichenforming fungus E. pusillum, whose genome has been sequenced [18], are analyzed in order to investigate the genetic mechanisms underlying drought resistance. In addition, representative genes that showed differential expressions in response to desiccation were further investigated in fresh lichen thallus E. pusillum during natural dehydration, to elucidate whether the pure-culture isolated mycobiont and the symbiotic mycobiont possess the same drought resistance mechanisms.

\section{Materials and methods}

\subsection{Materials and stress treatments}

The mycobiont of E. pusillum was isolated from the speci- men Z07020, originally collected from Shapotou Region of Ningxia, south-eastern edge of the Tengger desert, China (latitude $37.40^{\circ} \mathrm{N}$, longitude $105.00^{\circ} \mathrm{E}$ ). The fungal isolate was grown on $1.5 \%$ water agar for 1-2 weeks, and then transferred to Potato Dextrose Broth (PDB) medium cultured at room temperature. The artificial desiccation treatment was performed on the isolated lichen-forming fungus using polyethylene glycol (PEG), which is a non-permeable osmolyte and can create a severe water deficit [23]. The PEG-induced drought stress was used to investigate the drought-resistant of plants and fungi [24,25]. The mycobiont was desiccated under the condition of cultured in the medium containing 20\% PEG for 3 weeks.

The natural dehydration treatment was performed on the lichen thalli E. pusillum collected from Shapotou Desert in the same location as specimen Z07020. Under laboratory conditions, the fresh samples were completely rehydrated in deionized water for $5 \mathrm{~min}$ and excess water on the surface of thallus was removed using filter paper. The samples were then incubated at room temperature to naturally dehydrate for $0,40,60,150$ and $240 \mathrm{~min}$. At the end of each treatment, the samples were frozen immediately in liquid nitrogen and RNAs were extracted, then reverse-transcribed into cDNA as the template of quantitative real-time PCR (qRT-PCR).

\section{2 cDNA library construction and DNA sequencing}

Total RNA was extracted using the Trizol (Invitrogen, USA) extraction method according to the manufacturer's protocol. Poly-A mRNA was isolated with oligo-dT-coupled beads from $40 \mu \mathrm{g}$ total RNA of each sample and then sheared, and the isolated RNA samples were used for first strand cDNA synthesis which was random hexamers and Superscript II reverse transcriptase. After end repair and addition of a 3'dA overhang, the cDNA was ligated to Illumina paired-end adapter oligo mix, and size selected to about $200 \mathrm{bp}$ fragments by gel purification. After 16 PCR cycles, the libraries were sequenced using Illumina GAIIx (Illumina, USA) and the paired-end sequencing module.

\subsection{Mapping assembly of the transcriptome and dif- ferential expression analysis}

After removing the adapter sequences, the reads were mapped to the E. pusillum genome using PASA [26] and ORFs were found from the PASA assembly. Augustus [27], GeneID [28], and GeneMark-ES [29] programs were used to predict the gene models for the genome, and then the gene models generated by EVM [30] were updated by PASA. All predicted gene models were subjected to Gene Ontology (GO) [31], EuKaryotic Orthologous Groups (KOG) [32], FunCat [33] and Kyoto Encyclopedia of Genes and Genomes (KEGG) database analysis [34].

Raw array data were normalized using the ARRAYSTAR software (Dnastar, USA), and we used 
NOISeq [35] statistical method to identify differentially expressed gene between normal and drought stress cultivations. As suggested by NOISeq authors, a gene is declared as differentially expressed one if the probability ( $P$ value) is higher than 0.8 .

\subsection{Quantitative RT-PCR analysis}

Total RNA of the lichen-forming fungus E. pusillum was extracted from cultured fungal mycelium and lichen thalli respectively. cDNA syntheses were carried out according to the protocol described in the manual of Reverse Transcriptome System (Promega, USA). Real-time PCR was carried out using ABI 7500 real time PCR system (Applied Biosystems, USA). The data were analyzed using the $2^{-\Delta C_{\mathrm{t}}}$ method [36].

\section{Results}

\subsection{Functional classification of the transcriptome of E. pusillum upon water stress}

In the present study, we found that 1781 genes (probability $>0.8$ ) were differentially expressed in the desiccation condition (20\% PEG) in E. pusillum when compared with no water stressed condition (0\% PEG). The differentially expressed genes are $19.18 \%$ of 9285 genes annotated in the $37.5 \mathrm{Mb}$ genome of E. pusillum [18]. Among the 1781 genes, 1004 were up-regulated and 777 were down-regulated. Among them, 620 genes (35\%) were annotated as having unknown functions.

Using the Blast2GO platform [37], we classified the differentially expressed genes with GO terms according to their functions, and we found that most abundant GO terms were distributed in biological process, molecular functions and cellular components (Figure 1). Genes classified in the categories of "intracellular component", "cellular metabolic process", "transferase activity", "primary metabolic process" and "macromolecule metabolic process" were significantly up-regulated. As expected, the GO term "membrane component" was significantly overrepresented among the down-regulated genes, consistent with the strong effect of desiccation stress on the integrity of membranes. Surprisingly, very few genes belonging to the category of "stress response" were differentially expressed, with only three genes up-regulated and four down-regulated in the total of 51 genes. This result suggests that genes involved in PEG-induced stress response in E. pusillum are different from other organisms; therefore, there are likely new mechanisms of drought resistance in lichens.

When the differentially expressed genes were mapped to the KEGG using the Blast2GO platform (Figure 2), we found that genes whose expression level changed most markedly $(P \leqslant 0.0001)$ were related to ribosome and oxidative phosphorylation. Genes in several pathways were in- duced, including vitamin B6 metabolism, synthesis and degradation of ketone bodies, terpenoid backbone biosynthesis, oxidative phosphorylation, steroid biosynthesis, pyrimidine metabolism, and purine metabolism. Among these pathways, over $30 \%$ of all genes in the first five pathways mentioned above were up-regulated. However, only a few genes were significantly repressed and no genes were significantly up-regulated in nitrogen and galactose metabolisms $(P \geqslant 0.1)$. Most of these metabolism pathways exhibited active responses under the desiccation treatment, especially the pathways involved in essential function of the cell, such as ribosome, oxidative phosphorylation, pyrimidine metabolism, and purine metabolism.

\subsection{The expression changes of gene commonly involved in stress responses in $E$. pusillum}

Continuous exposure to drought leads to oxidative stress and induces defense mechanisms to scavenge the ROS that may result in significant damage to cell structure, it also induces osmotic pressure of cells and cells need to regulate this pressure by accumulating osmolytes [38]. Moreover, the osmolyte accumulation under drought can lead to misfolding and aggregation of proteins [39], and these changes can potentially limit cells' responses to desiccation [40]. Therefore, to better understand the drought resistant mechanism in E. pusillum, we investigated the expression changes of genes commonly involved in the response to stresses in other organisms, such as oxidative stress, osmotic regulation and post-translational processing under our drought treatment.

Previous research has revealed that oxidative defense mechanism in other organisms involved a number of specific enzymes, such as superoxide dismutase (SOD), catalase, peroxidases and auxiliary enzymes; and lowmolecular-weight antioxidants, such as tripeptide glutathione (GSH) and ascorbate [16,41,42]. In this study, three genes encoding antioxidation enzymes were induced in the E. pusillum genome under PEG-induced drought stress, including one for SOD and two for peroxidase (Table S1 in Supporting Information). By comparison, more genes involved in low-molecular-weight antioxidants were induced in E. pusillum, including those coding for thioredoxin, glutathione, and vitamin B6 (Table S1 in Supporting Information). We identified seven highly expressed thioredoxinlike genes, whose expressions were significantly upregulated in E. pusillum under desiccation. In addition, five genes related to glutathione S-transferase (GST), which were involved in glutathione metabolism, were up-regulated under desiccation in E. pusillum. This result indicates that $E$. pusillum regulates the glutathione metabolism in response to drought-induced oxidative stress. To our surprise, among the three genes for trehalose-6-phosphate synthase in $E$. pusillum (Table $\mathrm{S} 2$ in Supporting Information), none of them is differentially expressed (probability<0.8). Similarly, 
Total gene number (\%)

Up-regulated gene number (\%)

Down-regulated gene number (\%)

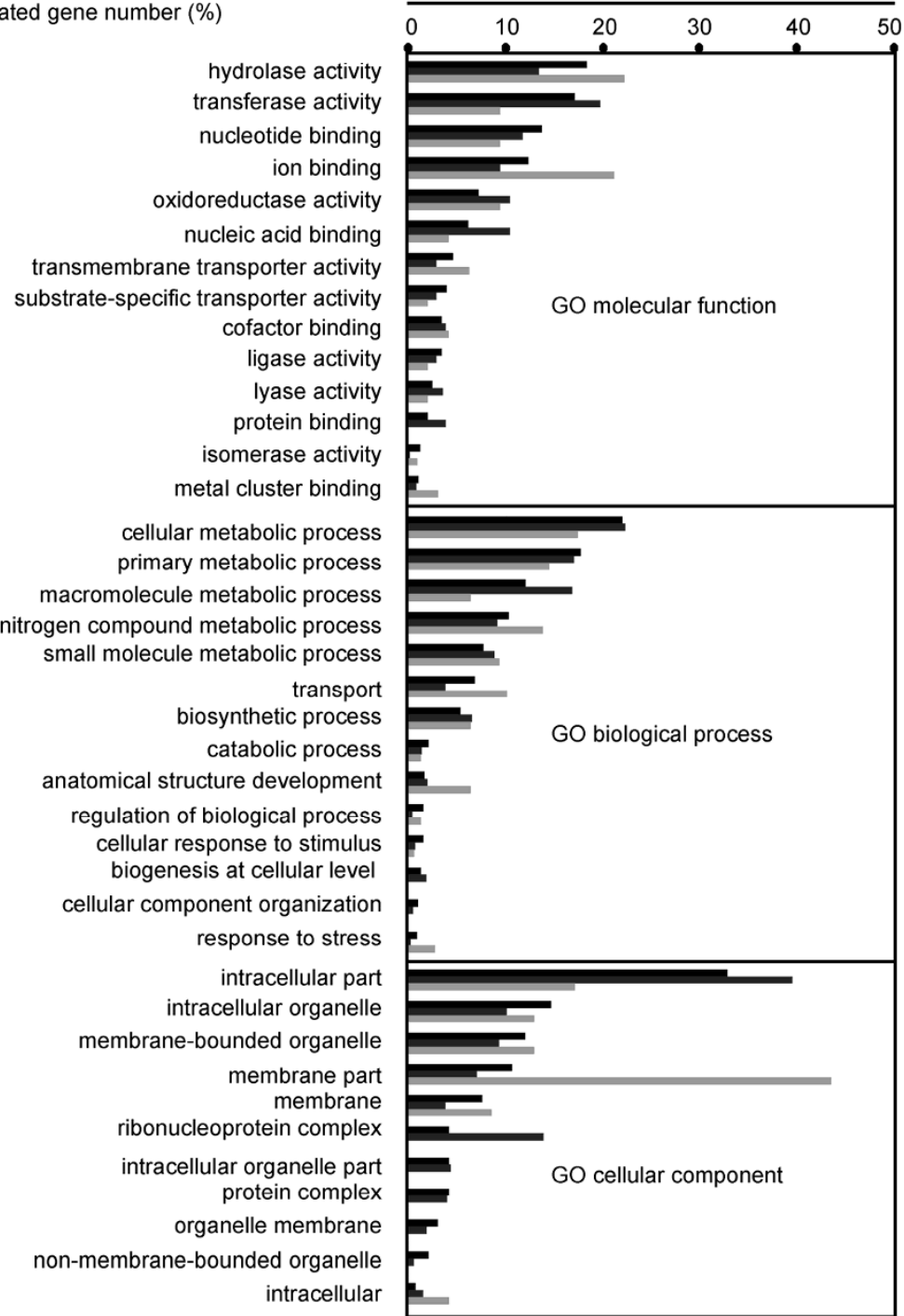

Figure 1 Gene Ontology classification of differentially expressed genes upon water stress in E. pusillum according to GO groups based on molecular function, biological process, and cellular component. The percentages of the total matched genes, up-and down-regulated genes contained in a particular GO group, which were labeled as black, dark grey, and light grey bars, respectively.

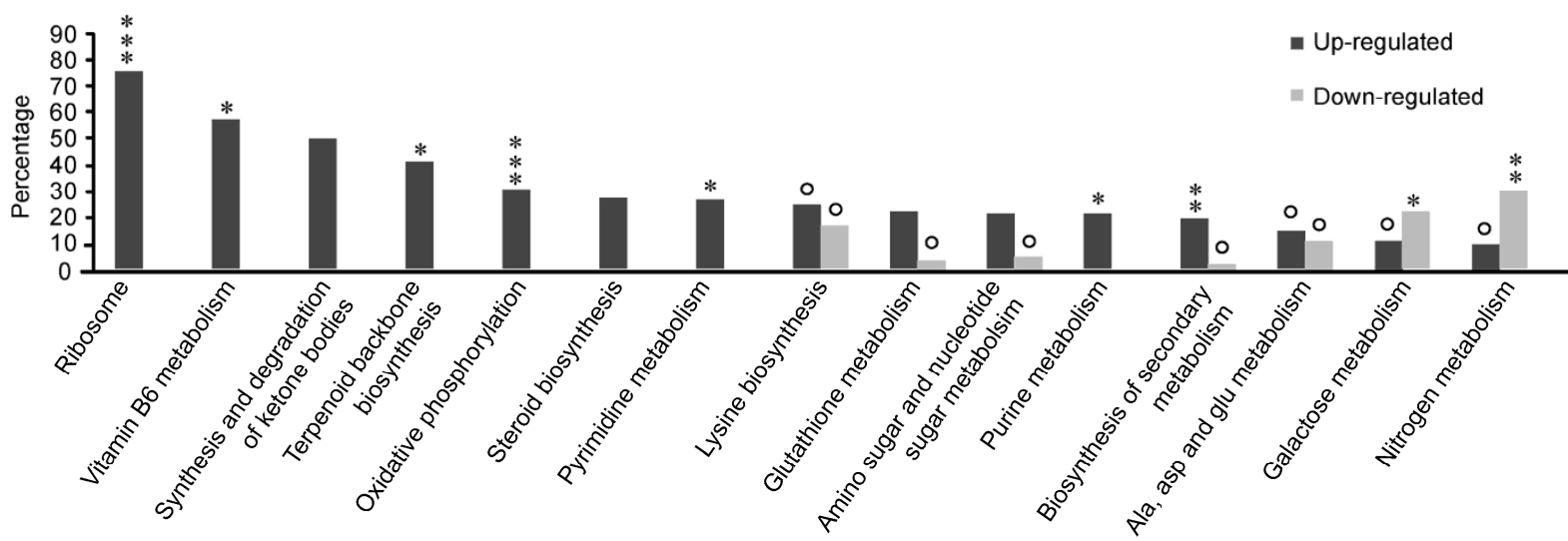

Figure 2 Functional classification of differentially expressed genes upon water stress in E. pusillum according to KEGG analysis. The percentage of upand down-regulated genes in a particular KEGG pathway was labeled as dark grey and light grey bars, respectively. Asterisks indicate the significance differences of differentially expressed genes. *, $P \leqslant 0.01 ; * *, P \leqslant 0.001 ; * * *, P \leqslant 0.0001 ;{ }^{\circ}, P \geqslant 0.1$. 
no other osmotic regulators were differentially expressed in E. pusillum under drought. However, we did identify 11 chaperone genes (including the heat shock protein (HSP), GroEL, and chaperonins) and 10 genes in proteasomes in $E$. pusillum (Table S3 in Supporting Information) to be significantly induced under PEG treatment.

Under stressed conditions, effective signal transduction is needed to communicate information from the external environment to the inside of a cell. Therefore, the expression of genes involved in signal transduction is expected to vary widely between normal and stress conditions. In this study, a total of 40 genes are annotated as related to signaling pathway in the transcriptome of E. pusillum, including calcium-mediated signaling, MAPK signaling pathway, TOR signaling pathway, mTOR signaling pathway, and G protein coupled receptor protein signaling pathway. However, only four genes (one for calcium-mediated signaling and three for MAPK signaling pathway) were differentially expressed (Table S4 in Supporting Information), and all four were down-regulated in E. pusillum under PEG-induced stress condition.

Transcription factors (TFs) function as key regulators of gene expression in response to environmental stresses. A total of 25 genes encoding putative TFs in E. pusillum genome were differentially expressed under PEG-induced stress. These 25 genes belonged to15 groups, i.e., zinc finger, TFIIA, heat shock, MADS, P53, NAC, DIP2, MYB, bZIP, Nus A, CBF, FOX, MEIS1, GATA, and Cmr1. Some of these TFs had been shown to function under abiotic stresses in plants and fungi [43-47]. For E. pusillum cultured under PEG-induced water limitations, seven of the 25 were up-regulated and 18 were down-regulated (Table S5 in Supporting Information). This result might be useful for the study of gene transcription regulation in lichen-forming fungi.

\subsection{Other differentially expressed genes revealed drought-adaptive mechanism of $E$. pusillum}

Except for genes commonly involved in the response to stresses in other organisms mentioned above, we identified some other genes that were differentially expressed in PEG-stressed E. pusillum from the transcriptome analysis (Figure 2). These differentially expressed genes can be classified into several categories: (i) Genes encode ribosomal proteins. The most significantly up-regulated transcripts are ribosomal proteins, and over $70 \%$ of ribosomal proteins were induced under PEG-induced drought. (ii) Genes involved in pyrimidine and purine metabolisms. The high transcriptional levels of these genes in stressed transcriptome (Table S6 in Supporting Information) indicate higher synthetic rates of DNA and RNA in E. pusillum under desiccation. (iii) Genes involved in oxidative phosphorylation. Oxidative phosphorylation is one of the pathways that showed the most significant differential expression of genes.
Specifically, genes for ATPase, NADH-ubiquinone oxidoreductase, Fe-S protein, and cytochrome $\mathrm{C}$ oxidase were all up-regulated under drought stress, and no gene in this pathway was down-regulated (Table S7 in Supporting Information). (iv) Genes involved in the nitrogen metabolisms. These genes were significantly repressed under desiccation (Table S8 in Supporting Information), which were marked in Figure 3. As a key compound in cellular metabolism, glutamate is synthesized using ammonium as a substrate through the functions of glutamate synthase and asparagine synthase. In this pathway, the generation of ammonium from formamide and nitroalkane is catalyzed by acetamidase/formamidase and 2-nitropropane dioxygenase. These enzymes were all repressed (Table S8 in Supporting Information), suggesting that the E. pusillum cells reduced the synthesis of glutamate through nitrogen metabolism under PEG-induced desiccation. However, the induced glutathione metabolism provides compensatory glutamate by the catalysis of glutathione S-transferase (Figure 3; Table S8 in Supporting Information). It has been reported that nitrogen metabolism was affected by some stressed conditions in other organisms $[48,49]$ although the mechanism is not clear. In this study, the expression changes of genes involved in nitrogen metabolism were likely the results of feedback inhibition of induced glutathione metabolism because the latter were known to play an important role under drought stress. (v) Genes involved in carbon metabolism, including nucleotide sugar metabolism and galactose metabolism (Table S8 in Supporting Information). Before galactose can be metabolized through the glycolysis pathway, it must be converted into glyceraldehydes-3P by galactokinase, galactose oxidase, and galactonate dehydratase (Figure 4). Among the differentially expressed genes in E. pusillum, those coding for galactose oxidase and galactonate dehydratase, which are indispensable enzymes in the galactose metabolism, were significantly down-regulated (Table

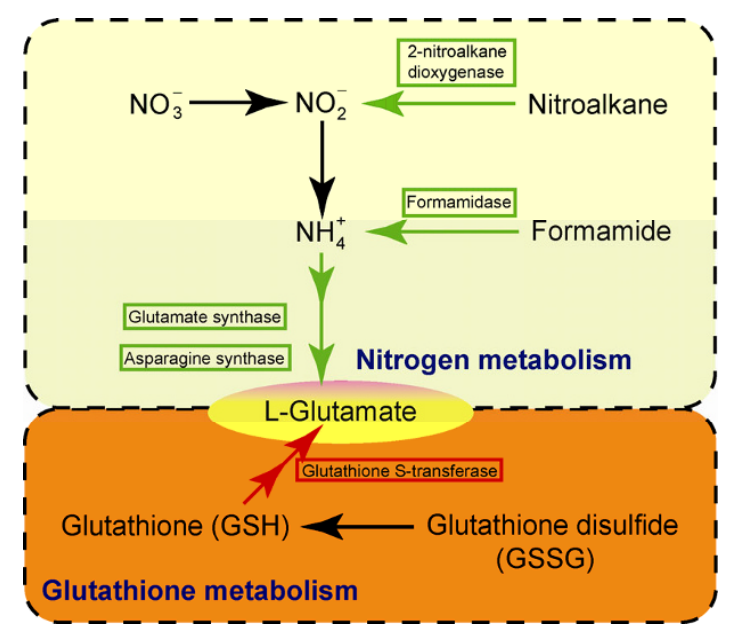

Figure 3 A diagram of nitrogen and glutathione metabolism, in which enzymes are encoded by genes either up- (labeled in red) or downregulated (labeled in green) by water stress in E. pusillum. 


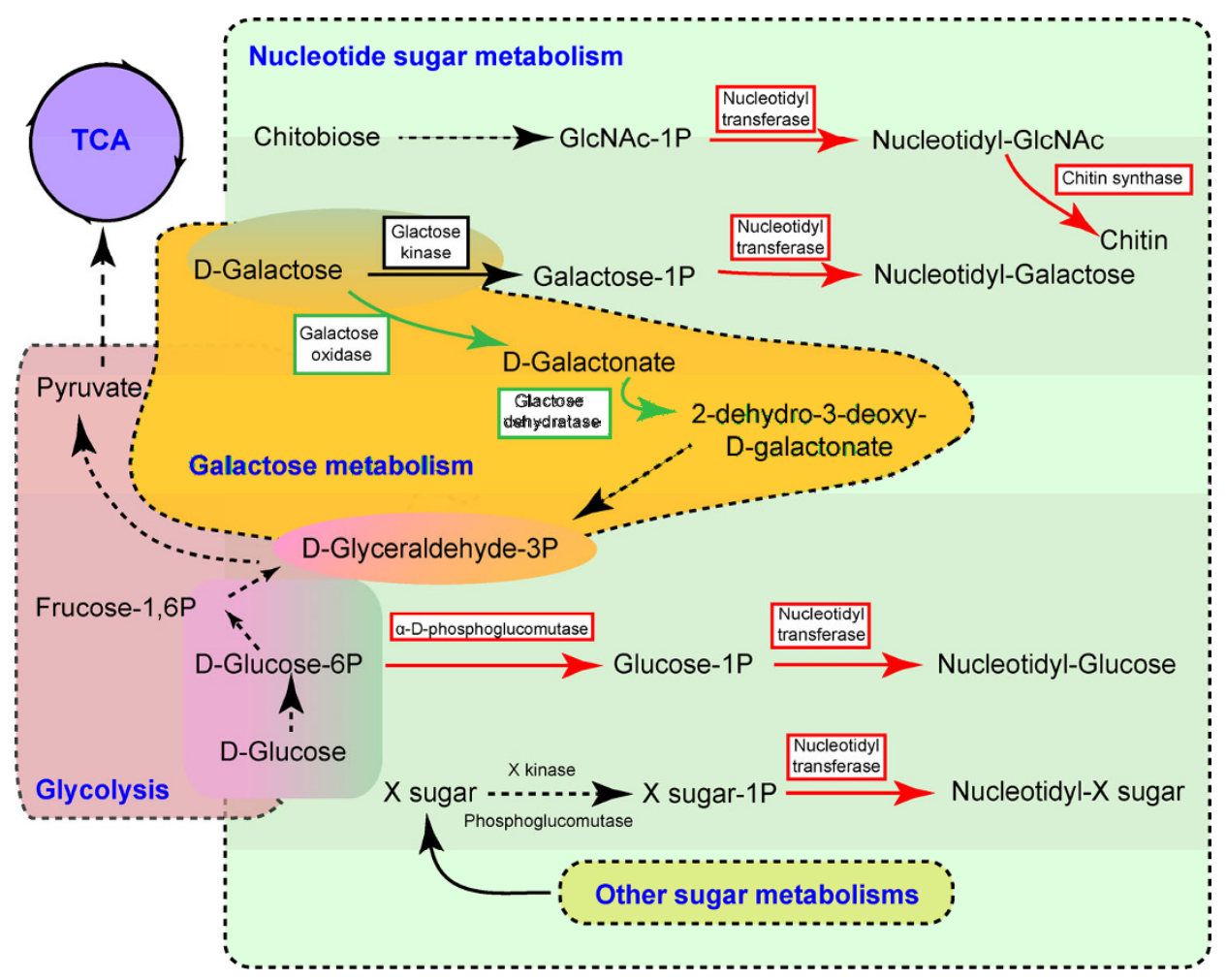

Figure 4 A diagram of carbon metabolism, including nucleotide sugar metabolism, galactose metabolism, and glycolysis, in which enzymes are encoded by genes either up- (labeled in red) or down-regulated (labeled in green) by water stress in E. pusillum.

S8 in Supporting Information). However, the accumulated galactose might be converted into nucleotide galactose through amino sugar and nucleotide metabolism. The common reaction in this metabolism is that sugars can be converted into nucleotide sugars by kinases, $\alpha$-D-phosphohexomutase, and nucleotidyltransferase. The expressions of the last two enzymes were up-regulated under drought stress (Table S8 in Supporting Information), which suggested that more nucleotide sugars were produced from monosaccharides and disaccharides in E. pusillum under desiccation. In addition, the up-regulation of the chitin synthase gene increases the amount of chitin, potentially making the cell wall thicker and preventing water loss and the damages caused by desiccation.

\section{4 qRT-PCR analysis of transcript levels for differen- tially expressed genes during dehydration process of lichen thallus}

Using qRT-PCR, a set of 23 representative drought response-related genes (Table 1) were selected to further monitor their transcript levels in E. pusillum thallus under natural dehydration stress. These genes are involved in posttranslational modification, osmotic regulation, energy providing, damage repairing (including ribosome protein, pyrimidine and purine metabolism), and carbon metabolism (including nucleotide sugar metabolism and galactose metabolism).
Because the degree of the stress for lichen thalli grown under natural conditions is difficult to determine, a dehydration process was performed for fresh lichen thalli of $E$. pusillum in laboratory to monitor the expression of lichen-forming fungal genes undergoing different degrees of drought stress. The lichen thalli were totally rehydrated, and the relative water content was detected over the dehydration process (Figure 5). Within the first $120 \mathrm{~min}$, the dehydration rate is fast and it did not exhibit significant change after $150 \mathrm{~min}$. The relative water content was close to zero after dehydration for $150 \mathrm{~min}$, which means that cells should have lost all the free water and are under severe drought stress at this time point. Here, the expressions of the selected genes

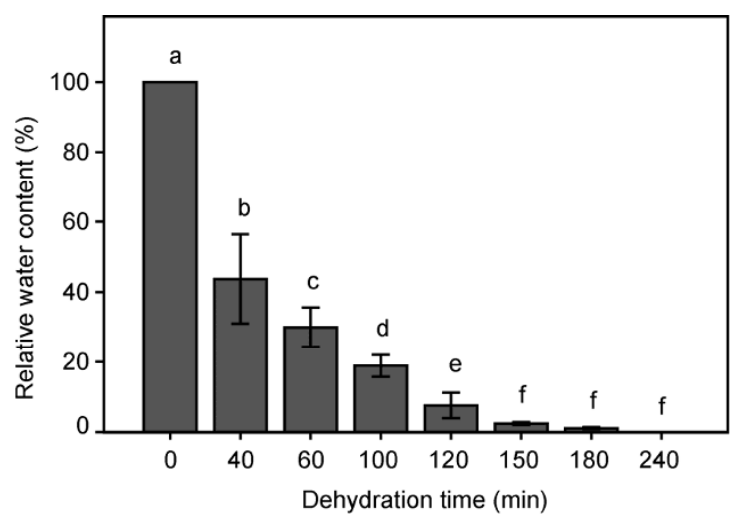

Figure 5 Relative water content in dehydrating lichen thalli of E. pusillum at different dehydration time period $(n=3)$. 
at this time point were set to 1 . A series of time points $(0,40$, 60,150 and $240 \mathrm{~min}$ ) were chosen to investigate the transcript levels of candidate genes, and the samples dehydrated for 150 and $240 \mathrm{~min}$ were regarded as samples under drought stress. For comparison of the relative expression at different time points, a heat-map was generated using the $\log _{2}$ fold change.

From the heat-map representation (Figure 6), the test genes can be classified into four classes according to the cluster analysis. In the first cluster (marked as blue), the

Table 1 The selected genes for qRT-PCR to verify the expression levels

\begin{tabular}{|c|c|c|c|c|}
\hline Term & Gene & Annotation & $\begin{array}{l}\text { Expression level } \\
\text { (stress) }\end{array}$ & $\begin{array}{c}\text { Expression level } \\
\text { (control) }\end{array}$ \\
\hline \multirow{2}{*}{ Ribosome } & F481_05528 & $40 \mathrm{~S}$ ribosomal protein $\mathrm{S} 3 \mathrm{Ae}$ & 739.98 & 193.08 \\
\hline & F481_05538 & Ribosomal protein S10 & 1380.97 & 257.81 \\
\hline \multirow{2}{*}{ Purine and pyrimidine metabolism } & F481_00996 & Nucleoside diphosphate kinase & 4654.23 & 209.72 \\
\hline & F481_02100 & dUTP pyrophosphatase & 108.25 & 17.32 \\
\hline Peroxidase & F481_03410 & Dyp-type peroxidase & 111.81 & 16.58 \\
\hline \multirow[t]{2}{*}{ Glutathione metabolism } & F481_02789 & $\begin{array}{l}\text { Glutathione S-transferase (GST), C-terminal do- } \\
\text { main }\end{array}$ & 227.39 & 69.51 \\
\hline & F481_06421 & Thioredoxin-like & 1736.00 & 241.98 \\
\hline \multirow{2}{*}{ Vitamin B6 metabolism } & F481_00322 & Pyridoxal 5'-phosphate (PLP) synthase & 1206.83 & 91.60 \\
\hline & F481_02092 & Pyridoxal kinase & 15.00 & 3.74 \\
\hline \multirow{3}{*}{ Oxidative phosphorylation } & F481_04003 & Complex $1 \mathrm{LYR}$ protein & 242.53 & 49.53 \\
\hline & F481_02166 & ATPase, F0 complex, subunit B, mitochondrial & 815.06 & 121.74 \\
\hline & F481_01557 & Cytochrome $\mathrm{c}$ oxidase, subunit VIb & 1274.81 & 329.34 \\
\hline Proteasome & F481_03276 & Proteasome, subunit beta type 3 & 552.61 & 69.64 \\
\hline \multirow{2}{*}{ Molecular chaperone } & F481_05773 & GroES-like & 1284.79 & 178.94 \\
\hline & F481_07753 & Heat shock protein $70 \mathrm{kD}$ & 314.87 & 63.86 \\
\hline Trehalose-6-phosohate synthase & F481_01316 & Trehalose-6-phosohate synthase & 61.13 & 37.70 \\
\hline \multirow{2}{*}{ Nitrogen metabolism } & F481_06302 & Glutamate synthase subunit alpha & 14.40 & 124.63 \\
\hline & F481_07075 & Nitritereductase & 3.41 & 158.61 \\
\hline \multirow{2}{*}{ Galactose metabolism } & F481_03176 & D-galactonate dehydratase & 10.09 & 159.01 \\
\hline & F481_05966 & Galactose oxidase, central domain & 7.79 & 31.83 \\
\hline \multirow{2}{*}{ Nucleotide sugar metabolism } & F481_07291 & Nucleotidyltransferases & 145.63 & 29.90 \\
\hline & F481_00358 & $\mathrm{N}$-acetyltransferase & 238.40 & 64.84 \\
\hline
\end{tabular}

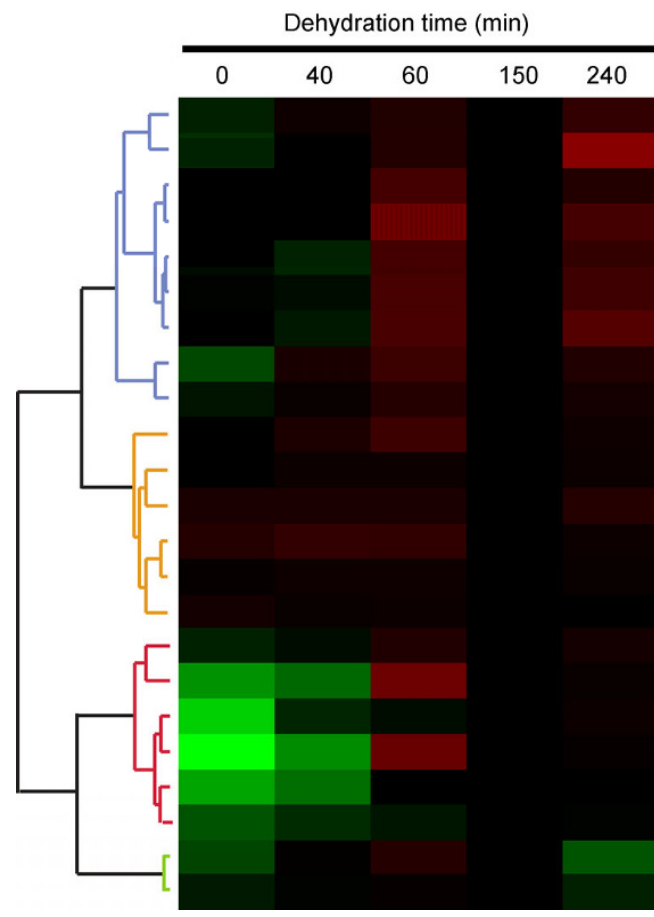

F481_02166-ATPase, F0 complex, subunit B

F481_01557-Cytochrome c oxidase, subunit Vib

F481_06421-Thioredoxin-like

F481_00358-N-acetyltransferase

F481_02789-Glutathione S-transferase

F481_07753-Heat shock protein $70 \mathrm{kD}$

F481_03176-D-galactonate dehydratase

F481_00322-Pyridoxal 5'-phosphate synthase

F481_07291-Nucleotidyltransferases

F481_05773-GroES-like

F481_03276-Proteasome, subunit beta type 3

F481 01316-Trehalose-6-phosohate synthase

F481_02100-dUTP pyrophosphatase

F481_04061-Proteasome, subunit alpha/beta

F481_00996-Nucleoside diphosphate kinase

F481_05528-40S ribosomal protein S3Ae

F481_05966-Galactose oxidase

F481_02092-Pyridoxal kinase

F481 03410-Dyp-type peroxidase

F481_04003-Complex 1 LYR protein

F481_05538-Ribosomal protein S10

F481_06302-Glutamate synthase subunit alpha

F481 07075-Nitrite reductase

Figure 6 Relative transcript levels of stress-related genes in E. pusillum under natural dehydration process. 
transcript levels of these genes increased during the first 60 min of dehydration, and were then significantly downregulated from 60 to $150 \mathrm{~min}$. A striking feature of these genes was that they were up-regulated at low water content from 150 to $240 \mathrm{~min}$, and the relative expressions at 240 min were higher than those at the beginning. The result indicated that the expressions of these genes were correlated to drought stress so they were likely to be related to drought-resistant mechanisms. Genes involved in antioxidation response mechanisms (vitamin B6 metabolism and glutathione), HSP, oxidative phosphorylation, and sugar metabolisms (nucleotide sugar metabolism and galactose metabolism) belonged to this class. In the second cluster (marked as yellow), the transcript levels of these genes do not exhibit significant fluctuations during the whole dehydration process, although the relative expressions of these genes at 150 min were slightly lower than those at other time points. Genes coding for proteins involved in purine and pyrimidine metabolism, proteasome, the trehalose-6-phosohate synthase (TPS), and GroES belonged to this class. At the first time point, the cells were undergoing long period drought and the expressions of these genes are not yet decreased dramatically, which suggests that these genes are highly expressed under both the wet and drought periods, therefore they seem to be house-keeping genes. In the third cluster (marked as red), the trend of gene expression during the first three time points is the same as that in the first cluster, but the up-regulation trend is more significant. The relative expressions of genes in this cluster at the last two time points were obviously higher than those at the first two or three time points, suggesting that these genes are highly expressed under drought; therefore, they can be regarded as drought-resistant related genes. Genes coding for ribosome, enzymes of galactose metabolism, and antioxidant mechanisms (vitamin B6 metabolism and peroxidase) belonged to this class. In the fourth cluster (marked as green), the relative expression during the five time points was present as a bell-shaped curve, with a significant declining at the low water level. The genes in this cluster are not the typical drought-resistant genes, such as those involved in nitrogen metabolism.

Generally, after rehydration for $60 \mathrm{~min}$, the relative expressions of these 23 selected genes reach a maximum, which implies that it needs to take some time to reflect non-drought state at transcript level in lichen-forming fungi. The results above indicate that the trends of expression of these drought-related genes vary widely. Genes in the first three clusters can be considered as drought-resistant genes, because they exhibited increased expression level when the water content was low after 150 min of dehydration. While the genes in the second cluster did not exhibit significant variation in their expressions, they nonetheless play very important roles under stressful conditions, because they are important housekeeping genes and their expressions are apt to be constant even under extreme desiccation.

\section{Discussion}

The model lichen-forming fungus E. pusillum was used to explore the drought stress response mechanisms in the present study. Our analyses identified 1781 differentially expressed genes, which are potential targets for further investigating the stress response mechanisms and characterizing the function of stress-related genes.

A large number of differentially expressed genes were classified into metabolism process (Figure 1), which suggests that E. pusillum had active metabolism under PEG-induced drought stress. This phenomenon is different from other drought-resistant organisms [50-52], where metabolic processes were largely suppressed during desiccation including PEG-induced stress. This result suggests that different from most other organisms, E. pusillum is a drought-adapted organism.

\subsection{The common drought-resistant mechanisms in $E$. pusillum}

The drought-resistant mechanisms, including reduce oxidative stress, osmotic regulation, and post-translational processing, in E. pusillum were compared to those in other drought-resistant plants and fungi. Our results show that $E$. pusillum has multiple mechanisms to deal with oxidative stress. Vitamin B6 has three forms (pyridoxine, pyridoxal, and pyridoxamine) and is an enzymatic cofactor participating in many biochemical reactions [53]. Recent research showed that vitamin B6 played a critical role in resistance to oxidative stress. For example, vitamin B6 is able to protect cells against cell death induced by ROS in the filamentous fungus Cercospora nicotianae [54]. Similarly, the loss of $p d x l$ gene which encodes a pyridoxine synthase in Arabidopsis thaliana can result in hypersensitive to osmotic stress and oxidative stress [55]. Therefore, it is not surprising that the expressions of genes involved in vitamin B6 biosynthesis in E. pusillum are significantly induced under desiccation (Table S1 in Supporting Information, Figure 2). Several antioxidant mechanisms, such as SOD and glutathione, have been reported in lichens [56]. However, this is the first report demonstrating that the low-molecular-weight antioxidant vitamin B6 is related to oxidative stress response and drought resistance in lichen-forming fungi.

Molecular chaperones direct protein folding and assembly, including refolding denatured proteins induced by stresses. There are many different families of chaperones, such as HSP, GroEL, and chaperonins. Each family acts to aid protein folding in different ways. For example, HSPs are induced mainly in response to high temperatures and other cellular stresses damages. In contrast, GroEL generally participates in response to the reduction in folding efficiency induced by macromolecule aggregation. The roles of proteasome are to degrade unnecessary or damaged proteins 
$[57,58]$, and it has been found that genes encoding proteasomes are up-regulated under stress [59,60]. The up-regulated expression of chaperone genes demonstrates that E. pusillum can likely cope with problems associated with protein misfolding and aggregation induced by desiccation through up-regulating its main chaperones. In addition, the increasing expression of proteasome implies that the misfolded proteins were likely quickly degraded [61].

At present, our knowledge of osmotic regulation is mainly established in plants [62], and a variety of osmolytes have been identified, including sugar, proline, malondialdehyde and potassium $[63,64]$. Among them, the sugar trehalose is the most important osmotic regulator in fungi $[65,66]$. No osmotic regulators were differentially expressed indicated that these ubiquitous osmotic regulators found in other organisms were not induced in E. pusillum under PEG-induced drought stress. Such a result would imply either that there are other osmotic regulators in E. pusillum, or that these known regulators are constitutively expressed in this lichen-forming fungus under both the normal and water-stressed conditions because of its drought adaptive character.

Recent studies have shown that an abiotic stress can trigger a set of common stress response signaling pathways in fungi, such as the cyclic AMP (cAMP) signaling pathway, $\mathrm{Ca}^{2+}$-dependent protein kinases (CDPKs), mitogen-activated protein kinases (MAPKs), protein kinase $\mathrm{C}$ (PKC), and Hog1 MAPK pathway [67-70]. One genes encoding for calcium-mediated signaling and three genes for MAPK signaling pathway were differentially expressed indicates that at least two signaling pathways participate in the drought response in lichen-forming fungus E. pusillum, and the functions of the differentially expressed genes are worthy of further investigation.

\subsection{Drought-adaptive mechanisms in E. pusillum}

In consideration of the strong drought tolerance characteristics of this lichen, we think that some different drought response mechanisms exist in E. pusillum. The ribosome is an intricate ribonucleo protein complex responsible for polypeptide synthesis in all living cells, so the expression changes of ribosomal proteins affect the translation process. Generally, the expressions of ribosomal proteins are regulated in response to environmental changes and cellular needs, such as nutrition increase, heat stress, and starvation (amino acid/nitrogen deprivation), with reduced expression of these proteins under stressful and starvation conditions [71-73]. The expressions of these proteins also change through the growth phases in a stationary culture, with increased expressions during the exponential growth phase and decreased expression during stationary phase. Recent research discovered that ribosomal subunits could act as the regulatory elements or filters which mediate interactions between particular mRNAs and components of the transla- tion machinery, thus providing a mechanism for translational control [74]. Therefore, it has been suggested that the increased expression of ribosomal proteins in E. pusillum could meet the demand of the expression of specific mRNAs required for stress response. Similarly, this phenomenon has also been found in other desiccation tolerant organisms [75-77]. The PEG-induced stress affects and accelerates the transcription and translation process, which can account for the active drought response mechanisms that more materials, such as nucleotides and amino acids, are needed to repair the DNA, RNA and protein damages caused by desiccation.

Almost all aerobic organisms need ATP to supply energy for metabolism, and oxidative phosphorylation is the dominating ATP synthesis pathway in mitochondrion. In other plants and fungi, the ATPase and ATP synthesis pathway have shown to be suppressed under different stress treatments [78,79]. However, KEGG assignment illustrates that most metabolic pathways were active in E. pusillum under PEG-induced stress (Figure 2). Our results suggest that $E$. pusillum, unlike other drought-sensitive organism, is more adapted to drought stress so its oxidative phosphorylation is also active under PEG-induced drought stress.

The differential expression of genes involved in galactose and nucleotide sugar metabolisms suggests that the changing carbohydrate metabolism of the mycobiont under desiccation likely leads to reduced monosaccharides and increased polysaccharides. Many previous studies have shown that carbohydrate metabolism is highly susceptible to desiccation [80-82], but most changes were linked to adjusting osmotic pressure. However, few studies have related nucleotide sugar metabolism to drought resistance. Nucleotide sugars are the universal sugar donors for the formation of polysaccharides, glycoproteins, proteoglycans, glycolipids, and glycosylated secondary metabolites [83], and enzymes involved in nucleotide sugar production are important because of the potential to manipulate the composition of cell walls through substrate level control [84]. It is known that glycosylated hormones and secondary metabolites play important roles in plant resistance against biotic and abiotic stresses [85,86]. In lichens, several lightabsorbing secondary metabolites produced by fungi have also shown to provide protection for their photosynthetic partners against photo damage [87]. Therefore, it can be argued that the increased expression of genes related to nucleotide sugar synthesis is related to drought-resistant in $E$. pusillum. However, further research is needed in order to identify if glycosylated secondary metabolites indeed provide protection for its algae.

In conclusion, these differentially expressed genes in $E$. pusillum can be summarized in damage repair, energy provision, and carbon metabolisms-provide protection according to their function. Together, these results suggest that $E$. pusillum is adapted to water limitations and drought. 


\subsection{The comparison of transcript levels for differen- tially expressed genes between PEG-stressed and dehy- drated E. pusillum}

The transcript levels of drought response-related genes were verified based on the lichen thalli dehydration experiment, together with data from comparative transcriptome analysis. The qRT-PCR result is consistent with that from RNA-seq data in which the expressions of these drought-resistant genes were up-regulated when lichen-forming fungus $E$. pusillum was cultured after adding $20 \%$ PEG. However, the expressions of genes involved in galactose metabolism were different between the RNA-seq data and the result from RT-PCR. This was likely due to the specific environmental treatment effect. During the dehydration performed in the laboratory, the photosynthetic partner provided very limited carbohydrates to lichen-forming fungus. In contrast, the mycobiont under PEG-induced drought stress was grown in PDB medium containing plenty of carbohydrates and other nutrients. Thus, the dehydration experiment actually included both drought and starvation stresses, but the sample used in transcriptome sequencing only experienced drought stress. Hence, the difference for genes involved in galactose metabolism reflects the variations in stresses. In summary, the drought-resistant and drought-adaptive mechanisms for the lichen-forming fungus E. pusillum under PEG-induced stress and for its lichen thalli under natural dehydration stress were essentially the same.

Our transcriptome analyses have identified many differentially expressed genes with unknown functions. These genes may be involved in novel drought response pathways or mechanisms to contribute to the strong drought resistance in E. pusillum. The detailed roles of these genes await further investigation.

This work was supported by the National Natural Science Foundation of China (31270018), the Knowledge Innovation Program of the Chinese Academy of Sciences (KSCX2-EW-J-6), and the State Key Laboratory of Crop Stress Biology for Arid Areas, Northwest A\&F University.

1 Brown D, Rapsch S, Beckett A, Ascaso C. The effect of desiccation on cell shape in the lichen Parmelia sulcata taylor. New Phytol, 1987, 105: 295-299

2 Bewley JD. Physiological-aspects of desiccation tolerance. Annu Rev Plant Phys, 1979, 30: 195-238

3 Oliver MJ, Derek Bewley J. Desiccation - tolerance of plant tissues: a mechanistic overview. Hortic Rev, 1997, 18: 171-213

4 Kappen L. Response to Extreme Environments. New York: Academic Press, 1973. 311-380

5 Aubert S, Juge C, Boisson AM, Gout E, Bligny R. Metabolic processes sustaining the reviviscence of lichen Xanthoria elegans (link) in high mountain environments. Planta, 2007, 226: 1287-1297

6 Kranner I. Glutathione status correlates with different degrees of desiccation tolerance in three lichens. New Phytol, 2002, 154: 451-460

7 Lange OL. Experimentell-Ökologische Untersuchungen an Flechten der Negev-Wüste. I. $\mathrm{CO}_{2}$-Gaswechsel von Ramalina maciformis (Del.) Bory unter kontrotlierten Bedingungen im Laboratorium. Flora, 1969, 158: 324-359
8 Ascaso C, Brown D, Rapsch S. The ultrastructure of the phycobiont of desiccated and hydrated lichens. Lichenologist, 1986, 18: 37-46

9 Kranner I, Cram WJ, Zorn M, Wornik S, Yoshimura I, Stabentheiner E, Pfeifhofer HW. Antioxidants and photoprotection in a lichen as compared with its isolated symbiotic partners. Proc Natl Acad Sci USA, 2005, 102: 3141-3146

10 Nash T, Reiner A, Demmig-Adams B, Kilian E, Kaiser W, Lange O. The effect of atmospheric desiccation and osmotic water stress on photosynthesis and dark respiration of lichens. New Phytol, 1990, 116: 269-276

11 Tuba Z, Csintalan Z, Proctor MCF. Photosynthetic responses of a moss, Tortula ruralis, ssp. ruralis, and the lichens Cladonia convoluta and C. Furcata to water deficit and short periods of desiccation, and their ecophysiological significance: a baseline study at present-day $\mathrm{CO}_{2}$ concentration. New Phytol, 1996, 133: 353-361

12 Calatayud A, Deltoro VI, Barreno E, Tascon SD. Changes in in vivo chlorophyll fluorescence quenching in lichen thalli as a function of water content and suggestion of zeaxanthin-associated photoprotection. Physiol Plantarum, 1997, 101: 93-102

13 Weissman L, Garty J, Hochman A. Characterization of enzymatic antioxidants in the lichen Ramalina lacera and their response to rehydration. Appl Environ Microb, 2005, 71: 6508-6514

14 França MB, Panek AD, Eleutherio ECA. The role of cytoplasmic catalase in dehydration tolerance of Saccharomyces cerevisiae. Cell Stress Chaperon, 2005, 10: 167

15 Jiang MY, Zhang JH. Water stress-induced abscisic acid accumulation triggers the increased generation of reactive oxygen species and up-regulates the activities of antioxidant enzymes in maize leaves. J Exp Bot, 2002, 53: 2401-2410

16 Kranner I, Birtic S. A modulating role for antioxidants in desiccation tolerance. Integr Comp Biol, 2005, 45: 734-740

17 Junttila S, Laiho A, Gyenesei A, Rudd S. Whole transcriptome characterization of the effects of dehydration and rehydration on Cladonia rangiferina, the grey reindeer lichen. BMC Genomics, 2013, 14: 870

18 Wang YY, Liu B, Zhang XY, Zhou QM, Zhang T, Li H, Yu YF, Zhang XL, Hao XY, Wang M, Wang L, Wei JC. Genome characteristics reveal the impact of lichenization on lichen-forming fungus Endocarpon pusillum Hedwig (Verrucariales, Ascomycota). BMC Genomics, 2014, 15: 34

19 Veerman J, Vasil'ev S, Paton GD, Ramanauskas J, Bruce D. Photoprotection in the lichen Parmelia sulcata: the origins of desiccation-induced fluorescence quenching. Plant Physiol, 2007, 145: 997-1005

20 Scheidegger C, Schroeter B, Frey B. Structural and functional processes during water vapour uptake and desiccation in selected lichens with green algal photobionts. Planta, 1995, 197: 399-409

21 Holder J, Wynn-Williams D, Rull Perez F, Edwards H. Raman spectroscopy of pigments and oxalates in situ within epilithic lichens: Acarospora from the Antarctic and Mediterranean. New Phytol, 2000, 145: $271-280$

22 Zhang T, Wei J. Survival analyses of symbionts isolated from Endocarpon pusillum Hedwig to desiccation and starvation stress. Sci China Life Sci, 2011, 54: 480-489

23 Lagerwer J, Eagle HE, Ogata G. Control of osmotic pressure of culture solutions with polyethylene glycol. Science, 1961, 133: 1486-1487

24 Caruso A, Chefdor F, Carpin S, Depierreux C, Delmotte FM, Kahlem G, Morabito D. Physiological characterization and identification of genes differentially expressed in response to drought induced by PEG 6000 in Populus canadensis leaves. J Plant Physiol, 2008, 165: 932-941

25 Vivas A, Marulanda A, Ruiz-Lozano JM, Barea JM, Azcon R. Influence of a Bacillus sp on physiological activities of two arbuscular mycorrhizal fungi and on plant responses to PEG-induced drought stress. Mycorrhiza, 2003, 13: 249-256

26 Haas BJ, Delcher AL, Mount SM, Wortman JR, Smith Jr RK, Hannick LI, Maiti R, Ronning CM, Rusch DB, Town CD. Improving the Arabidopsis genome annotation using maximal transcript alignment 
assemblies. Nucleic Acids Res, 2003, 31: 5654-5666

27 Stanke M, Morgenstern B. Augustus: a web server for gene prediction in eukaryotes that allows user-defined constraints. Nucleic Acids Res, 2005, 33: W465-467

28 Parra G, Blanco E, Guigó R. Geneid in Drosophila. Genome Res, 2000, 10: 511-515

29 Ter-Hovhannisyan V, Lomsadze A, Chernoff YO, Borodovsky M. Gene prediction in novel fungal genomes using an ab initio algorithm with unsupervised training. Genome Res, 2008, 18: 1979-1990

30 Haas BJ, Salzberg SL, Zhu W, Pertea M, Allen JE, Orvis J, White O, Buell CR, Wortman JR. Automated eukaryotic gene structure annotation using EVidenceModeler and the program to assemble spliced alignments. Genome Biol, 2008, 9: R7

31 Ashburner M, Ball CA, Blake JA, Botstein D, Butler H, Cherry JM, Davis AP, Dolinski K, Dwight SS, Eppig JT, Harris MA, Hill DP, Issel-Tarver L, Kasarskis A, Lewis S, Matese JC, Richardson JE, Ringwald M, Rubin GM, Sherlock G, Consortium GO. Gene ontology: tool for the unification of biology. Nat Genet, 2000, 25: 25-29

32 Sonnhammer ELL, Koonin EV. Orthology, paralogy and proposed classification for paralog subtypes. Trends Genet, 2002, 18: 619-620

33 Ruepp A, Zollner A, Maier D, Albermann K, Hani J, Mokrejs M, Tetko I, Guldener U, Mannhaupt G, Munsterkotter M, Mewes HW. The funcat, a functional annotation scheme for systematic classification of proteins from whole genomes. Nucleic Acids Res, 2004, 32: 5539-5545

34 Kanehisa M, Goto S, Kawashima S, Okuno Y, Hattori M. The KEGG resource for deciphering the genome. Nucleic Acids Res, 2004, 32: D277-280

35 Tarazona S, García-Alcalde F, Dopazo J, Ferrer A, Conesa A. Differential expression in RNA-Seq: a matter of depth. Genome Res, 2011, 21: 2213-2223

36 Schmittgen TD, Livak KJ. Analyzing real-time PCR data by the comparative $C_{\mathrm{t}}$ method. Nat Protoc, 2008, 3: 1101-1108

37 Gotz S, Garcia-Gomez JM, Terol J, Williams TD, Nagaraj SH, Nueda MJ, Robles M, Talon M, Dopazo J, Conesa A. High-throughput functional annotation and data mining with the Blast2GO suite. Nucleic Acids Res, 2008, 36: 3420-3435

38 Bray EA. Molecular responses to water deficit. Plant Physiol, 1993, 103: 1035-1040

39 Ben-Zvi AP, Goloubinoff P. Review: mechanisms of disaggregation and refolding of stable protein aggregates by molecular chaperones. $\mathrm{J}$ Struct Biol, 2001, 135: 84-93

40 Welch AZ, Gibney PA, Botstein D, Koshland DE. TOR and RAS pathways regulate desiccation tolerance in Saccharomyces cerevisiae. Mol Biol Cell, 2013, 24: 115-128

41 Nyska A, Kohen R. Oxidation of biological systems: oxidative stress phenomena, antioxidants, redox reactions, and methods for their quantification. Toxicol Pathol, 2002, 30: 620-650

42 Navrot N, Rouhier N, Gelhaye E, Jacquot JP. Reactive oxygen species generation and antioxidant systems in plant mitochondria. Physiol Plantarum, 2007, 129: 185-195

43 Tamai KT, Liu XD, Silar P, Sosinowski T, Thiele DJ. Heat-shock transcription factor activates yeast metallothionein gene-expression in response to heat and glucose starvation via distinct signaling pathways. Mol Cell Biol, 1994, 14: 8155-8165

44 Damveld RA, Arentshorst M, Franken A, vanKuyk PA, Klis FM, van den Hondel CAMJJ, Ram AFJ. The Aspergillus niger MADS-box transcription factor RlmA is required for cell wall reinforcement in response to cell wall stress. Mol Microbiol, 2005, 58: 305-319

45 Guo M, Chen Y, Du Y, Dong YH, Guo W, Zhai S, Zhang HF, Dong SM, Zhang ZG, Wang YC, Wang P, Zheng XB. The bZIP transcription factor MoAP1 mediates the oxidative stress response and is critical for pathogenicity of the rice blast fungus Magnaporthe oryzae. Plos Pathog, 2011, 7: e1001302

46 Voitsik AM, Muench S, Deising HB, Voll LM. Two recently duplicated maize NAC transcription factor paralogs are induced in response to Colletotrichum graminicola infection. BMC Plant Biol,
2013, 13: 85

47 Nicholls S, Straffon M, Enjalbert B, Nantel A, Macaskill S, Whiteway M, Brown AJP. Msn2-and Msn4-like transcription factors play no obvious roles in the stress responses of the fungal pathogen Candida albicans. Eukaryot Cell, 2004, 3: 1111-1123

48 Debouba M, Maâroufi-Dghimi H, Suzuki A, Ghorbel MH, Gouia H. Changes in growth and activity of enzymes involved in nitrate reduction and ammonium assimilation in tomato seedlings in response to $\mathrm{NaCl}$ stress. Ann Bot, 2007, 99: 1143-1151

49 Planchet E, Rannou O, Ricoult C, Boutet-Mercey S, Maia-Grondard A, Limami AM. Nitrogen metabolism responses to water deficit act through both abscisic acid (ABA)-dependent and independent pathways in Medicago truncatula during post-germination. J Exp Bot, 2011, 62: 605-615

50 Dittami SM, Scornet D, Petit JL, Segurens B, Da Silva C, Corre E, Dondrup M, Glatting KH, Konig R, Sterck L, Rouze P, Van de Peer Y, Cock JM, Boyen C, Tonon T. Global expression analysis of the brown alga Ectocarpus siliculosus (Phaeophyceae) reveals large-scale reprogramming of the transcriptome in response to abiotic stress. Genome Biol, 2009, 10: R66

51 Foito A, Byrne SL, Shepherd T, Stewart D, Barth S. Transcriptional and metabolic profiles of Lolium perenne L. Genotypes in response to a PEG-induced water stress. Plant Biotechnol J, 2009, 7: 719-732

52 Gulez G, Dechesne A, Workman CT, Smets BF. Transcriptome dynamics of Pseudomonas putida KT2440 under water stress. Appl Environ Microbiol, 2012, 78: 676-683

53 Mooney S, Leuendorf JE, Hendrickson C, Hellmann H. Vitamin B6: a long known compound of surprising complexity. Molecules, 2009, 14: 329-351

54 Bilski P, Li M, Ehrenshaft M, Daub M, Chignell C. Vitamin B6 (pyridoxine) and its derivatives are efficient singlet oxygen quenchers and potential fungal antioxidants. Photochem Photobiol, 2000, 71: 129-134

55 Chen H, Xiong L. Pyridoxine is required for post-embryonic root development and tolerance to osmotic and oxidative stresses. Plant J, 2005, 44: 396-408

56 Kranner I, Beckett R, Hochman A, Nash TH. Desiccation-tolerance in lichens: a review. Bryologist, 2008, 111: 576-593

57 Grune T, Reinheckel T, Davies K. Degradation of oxidized proteins in mammalian cells. FASEB J, 1997, 11: 526-534

58 Sassa H, Oguchi S, Inoue T, Hirano H. Primary structural features of the 20S proteasome subunits of rice Oryza sativa. Gene, 2000, 250: 61-66

59 Wu P, Wang X, Qin GX, Liu T, Jiang YF, Li MW, Guo XJ. Microarray analysis of the gene expression profile in the midgut of silkworm infected with cytoplasmic polyhedrosis virus. Mol Biol Rep, 2011, 38: 333-341

60 Rajjou L, Belghazi M, Huguet R, Robin C, Moreau A, Job C, Job D. Proteomic investigation of the effect of salicylic acid on Arabidopsis seed germination and establishment of early defense mechanisms. Plant Physiol, 2006, 141: 910-923

61 Huang H, Moller IM, Song SQ. Proteomics of desiccation tolerance during development and germination of maize embryos. J Proteomics, 2012, 75: 1247-1262

62 Chaves MM, Maroco JP, Pereira JS. Understanding plant responses to drought-from genes to the whole plant. Funct Plant Biol, 2003, 30: 239-264

63 Shao HB, Liang ZS, Shao MA. Osmotic regulation of 10 wheat (Triticum aestivum L.) genotypes at soil water deficits. Colloid Surface B, 2006, 47: 132-139

64 Ashraf M, Foolad M. Roles of glycine betaine and proline in improving plant abiotic stress resistance. Environ Exp Bot, 2007, 59: 206-216

65 Hounsa CG, Brandt EV, Thevelein J, Hohmann S, Prior BA. Role of trehalose in survival of Saccharomyces cerevisiae under osmotic stress. Microbiology, 1998, 144: 671-680

66 Ashraf M. Inducing drought tolerance in plants: recent advances. Bi- 
otechnol Adv, 2010, 28: 169-183

67 Bahn YS, Xue C, Idnurm A, Rutherford JC, Heitman J, Cardenas ME. Sensing the environment: lessons from fungi. Nat Rev Microbiol, 2007, 5: 57-69

68 Alonso-Monge R, Roman E, Arana DM, Pla J, Nombela C. Fungi sensing environmental stress. Clin Microbiol Infec, 2009, 15: 17-19

69 Fernandes L, Araujo MA, Amaral A, Reis VC, Martins NF, Felipe MS. Cell signaling pathways in Paracoccidioides brasiliensis - inferred from comparisons with other fungi. Genet Mol Res, 2005, 4: 216-231

70 Brown SM, Campbell LT, Lodge JK. Cryptococcus neoformans, a fungus under stress. Curr Opin Microbiol, 2007, 10: 320-325

71 Li B, Nierras CR, Warner JR. Transcriptional elements involved in the repression of ribosomal protein synthesis. Mol Cell Biol, 1999, 19: 5393-5404

72 Warner JR. Synthesis of ribosomes in Saccharomyces cerevisiae. Microbiol Mol Biol Rev, 1989, 53: 256-271

73 Warner JR. The economics of ribosome biosynthesis in yeast. Trends Biochem Sci, 1999, 24: 437-440

74 Mauro VP, Edelman GM. The ribosome filter hypothesis. Proc Natl Acad Sci USA, 2002, 99: 12031-12036

75 Adhikari BN, Wall DH, Adams BJ. Desiccation survival in an Antarctic nematode: molecular analysis using expressed sequenced tags. BMC Genomics, 2009, 10: 69

76 Angelovici R, Galili G, Fernie AR, Fait A. Seed desiccation: a bridge between maturation and germination. Trends Plant Sci, 2010, 15: 211-218

77 Pearson GA, Hoarau G, Lago-Leston A, Coyer JA, Kube M, Reinhardt R, Henckel K, Serrao ET, Corre E, Olsen JL. An expressed sequence tag analysis of the intertidal brown seaweeds Fucus serratus
(L.) and F. vesiculosus (L.) (Heterokontophyta, Phaeophyceae) in response to abiotic stressors. Mar Biotechnol, 2010, 12: 195-213

78 Piper PW. The heat-shock and ethanol stress responses of yeast exhibit extensive similarity and functional overlap. FEMS Microbiol Lett, 1995, 134: 121-127

79 Tezara W, Mitchell VJ, Driscoll SD, Lawlor DW. Water stress inhibits plant photosynthesis by decreasing coupling factor and ATP. Nature, 1999, 401: 914-917

80 Toldi O, Tuba Z, Scott P. Vegetative desiccation tolerance: is it a goldmine for bioengineering crops? Plant Sci, 2009, 176: 187-199

81 Valliyodan B, Nguyen HT. Understanding regulatory networks and engineering for enhanced drought tolerance in plants. Curr Opin Plant Biol, 2006, 9: 189-195

82 Abdel Latef AAH. Influence of arbuscular mycorrhizal fungi and copper on growth, accumulation of osmolyte, mineral nutrition and antioxidant enzyme activity of pepper (Capsicum annuum L.). Mycorrhiza, 2011, 21: 495-503

83 Strominger JL. Nucleotide intermediates in the biosynthesis of heteropolymeric polysaccharides. Biophys J, 1964, 4: 139-153

84 Gibeaut DM. Nucleotide sugars and glycosyltransferases for synthesis of cell wall matrix polysaccharides. Plant Physiol Bioch, 2000, 38: 69-80

85 Roberts MR, Warner SA, Darby R, Lim EK, Draper J, Bowles DJ. Differential regulation of a glucosyl transferase gene homologue during defence responses in tobacco. J Exp Bot, 1999, 50: 407-410

86 O'Donnell PJ, Truesdale MR, Calvert CM, Dorans A, Roberts MR, Bowles DJ. A novel tomato gene that rapidly responds to wound- and pathogen-related signals. Plant J, 1998, 14: 137-142

87 Gauslaa Y, Solhaug KA. Fungal melanins as a sun screen for symbiotic green algae in the lichen Lobaria pulmonaria. Oecologia, 2001, 126: $462-471$

Open Access This article is distributed under the terms of the Creative Commons Attribution License which permits any use, distribution, and reproduction in any medium, provided the original author(s) and source are credited.

\section{Supporting Information}

Table S1 The differential expression of genes putatively involved in antioxidant mechanisms

Table S2 The differential expression of genes putatively involved in osmotic regulation

Table S3 The differential expression of genes putatively involved in post-translational processing

Table S4 The differential expression of genes putatively involved in signal transduction pathway

Table S5 The differential expression of genes putatively involved in transcriptional factor

Table S6 The differential expression of genes putatively involved in pyrimidine and purine metabolism

Table S7 The differential expression of genes putatively involved in oxidative phosphorylation

Table S8 The differential expression of genes putatively involved in nitrogen and carbon metabolism

The supporting information is available online at life.scichina.com and link.springer.com. The supporting materials are published as submitted, without typesetting or editing. The responsibility for scientific accuracy and content remains entirely with the authors. 